\title{
Upregulation of miR150-5p in generalized myasthenia gravis patients is associated with decreased serum levels of IL-17 and increased serum levels of IL-10
}

\author{
Wenling Ao, Canhui Tian, Xiongjun He, Yeli Hu, Weidong Wang, Yajie Liu
}

\begin{abstract}
Background. MiR150-5p has been reported to be involved in generalized myasthenia gravis, in which different cytokines play critical roles. The regulatory network of cytokines in generalized myasthenia gravis has not been fully elucidated. Our study aimed to investigate the interactions between miR150-5p and different cytokines in generalized myasthenia gravis.
\end{abstract}

Materials and Method. Serum levels of miR150-5p and different cytokines including IL-2, IL-17, IL-10, IL-19, IL-20 and IL-35 were detected by qRT-PCR and ELISA, respectively. ROC curve analysis was performed to evaluate the diagnostic value of miR150-5p for generalized myasthenia gravis. Correlation between serum levels of miR150-5p and different cytokines were analyzed by Pearson correlation coefficient.

Results. Compared with healthy controls, decreased serum levels of IL-2 and IL-17 and increased serum levels of miR150-5p, IL-10, IL-19, IL-20 and IL-35 were observed in patients with generalized myasthenia gravis. Serum levels of miR150-5p were positively correlated with IL-10 and negatively correlated with IL-17. After treatments, serum levels of miR150-5p and IL-10 decreased, while serum levels of IL-2 and IL-17 increased.

Conclusion. Upregulation of miR150-5p is involved in generalized myasthenia gravis patients and is associated with decreased serum levels of IL-17 as well as increased serum levels of IL-10.

Key words: generalized myasthenia gravis, MiR150-5p, cytokines

Received: July 13, 2018; Accepted: March 5, 2019; Available online: March 29, 2019

https://doi.org/10.5507/bp.2019.009

(C) 2020 The Authors; https://creativecommons.org/licenses/by/4.0/

Department of Neurology, Shenzhen Hospital, Southern Medical University, Shenzhen City, 518000, PR. China Corresponding author: Yajie Liu, e-mail: upvduf1@163.com

\section{INTRODUCTION}

Myasthenia gravis is an autoimmune disease with muscle weakness and fatigue as the main clinical symptoms ${ }^{1}$. Myasthenia gravis is caused by antibodies against proteins at the neuromuscular junction and fatigable weakness in skeletal muscles will be induced by disturbed neuromuscular transmission ${ }^{2}$. Thymic abnormalities are frequently observed in patients with early onset of myasthenia gravis $^{3}$. The thymic abnormalities have been proved to be mediated by overexpression of interferon (IFN)- $\beta$ in the myasthenia gravis thymus ${ }^{4}$. Although a variety of types of drugs have been developed to treat myasthenia gravis, prognosis of patients with this disease is still not satisfactory, possibly due to the lack of medication standard and complex pathogenesis ${ }^{5}$.

MicroRNAs (miRNAs) is a group of non-coding RNAs composed about 22 nucleotides, which is widely distributed across species and plays pivotal roles in RNA silencing and gene expression regulation at post-transcriptional level ${ }^{6,7}$. Previous studies have proved that miRNAs have critical functions in both physiological process and pathological changes ${ }^{8}$, including the development and progression of myasthenia gravis ${ }^{9}$. Development of myasthenia gravis is accompanied with changes in expression pattern of a series of inflammatory cytokines ${ }^{10}$. Those cy- tokines participate in the pathogenesis of myasthenia gravis through immune regulation ${ }^{11}$. A recent study showed that upregulation of miR-150-5p is likely to be involved in myasthenia gravis ${ }^{12}$, while the molecular mechanism is unknown. In the present study we further confirmed the involvement of miR-150-5p in myasthenia gravis and proved evidences that the involvement of miR-150-5p in myasthenia gravis is possibly mediated through its interactions with inflammatory cytokine networks.

\section{MATERIALS AND METHODS}

\section{Patients}

A total of 155 patients with generalized myasthenia gravis were diagnosed and treated in Shenzhen Hospital from January 2015 to January 2018. All patients were diagnosed according to the China guidelines for the diagnosis and treatment of generalized myasthenia gravis ${ }^{13}$. There were 34 cases in MGFA class 1, 18 in class 2a, 33 in class $2 \mathrm{~b}, 18$ in class $3 \mathrm{a}, 12$ in class $3 \mathrm{~b}, 21$ in class $4 \mathrm{a}$, 9 in $4 \mathrm{~b}$ and 10 in class V. Our study included 30 out of those patients according to the inclusion and exclusion criteria. Inclusion criteria are as follows: 1) patients diagnosed as generalized myasthenia gravis; 2) patients that completed treatment in our hospital; 3 ) patients who were 
A

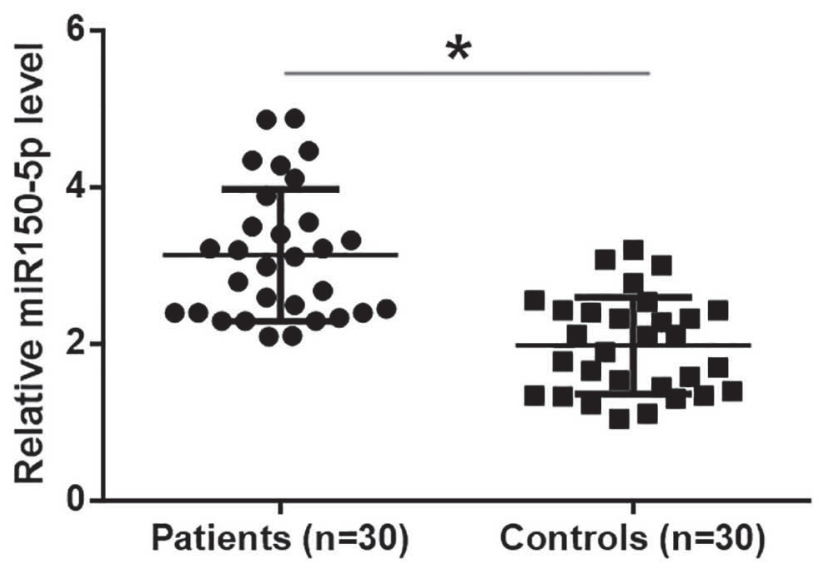

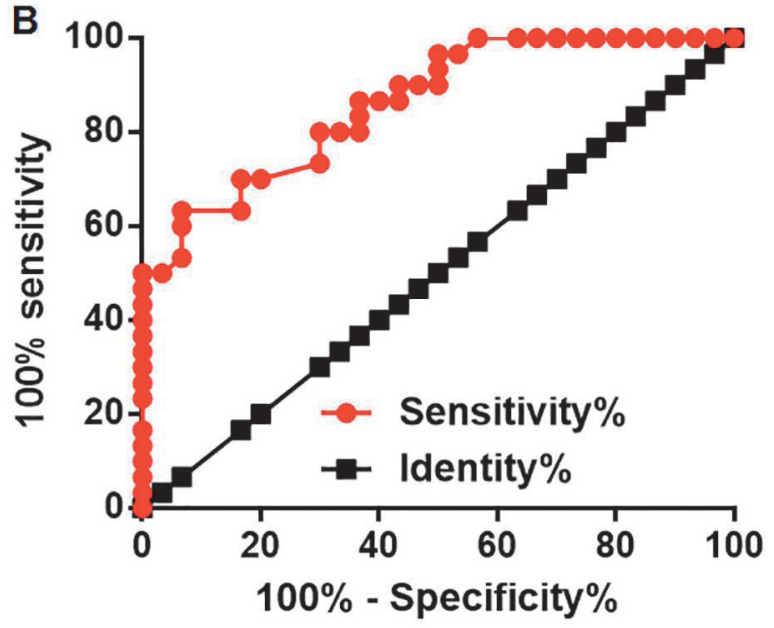

Fig. 1. Upregulation of miR150-5p distinguished generalized myasthenia gravis patients from healthy controls.

Serum levels of miR150-5p were significantly higher in generalized myasthenia gravis patients than in healthy controls (A) and upregulation of miR150-5p distinguished generalized myasthenia gravis patients from healthy controls $(\mathrm{B})\left({ }^{*}, P<0.05\right)$.

willing to join the study. Exclusion criteria are: 1) patients complicated with other severe or chronic diseases; 2) patients who were treated before the admission; 3 ) patients transferred to other hospital during treatment. Those patients included 18 males and 12 females, and age ranged from 22 to 63 years, with a mean age of $41.4 \pm 6.2$ years. At the same time, a total of 30 volunteers with normal physiological conditions were also included to serve as a control group. The control group included 17 males and 13 females, and the age ranged from 24 to 62 years, with a mean age of $40.9 \pm 5.8$ years. No significant differences in age and gender were found between two groups. The Ethics committee of Shenzhen Hospital approved this study. All participants signed the informed consent.

\section{Treatment}

All patients were treated with acetylcholinesterase inhibitors combined with glucocorticoid and other immunosuppressants, e.g. azathioprine, cyclosporine, tacrolimus and MMF. Intravenous immunoglobulin (IVIg) was also used. Medication was reduced or stopped several months (> 3 months) after the signs of myasthenia gravis improved or disappeared. Clinical symptoms disappeared in all patients and no recurrence occurred within 1 month after treatment. No significant adverse reactions were observed.

\section{Blood extraction and serum preparation}

Blood $(10 \mathrm{~mL})$ was extracted from the elbow vein of both patients and healthy controls on the day of admission. Blood was also extracted from patients on the day of discharge. Blood was kept at room temperature for 30 min, followed by centrifugation at $1000 \mathrm{~g}$ for $20 \mathrm{~min}$ to collected serum. Serum was stored in liquid nitrogen before use.

\section{RNA insolation and real-time quantitative reverse transcription PCR (qRT-PCR)}

MiRNA was extracted from serum using TaqMan miRNA Isolation kit (Applied Biosystems, Foster City, CA, USA). Reverse transcription was performed using
TaqMan MicroRNA Reverse Transcription Kit (Thermo Fisher Scientific). PCR reaction was performed to detect miR150-5p expression using mirVana qRT-PCR miRNA Detection Kit (Thermo Fisher Scientific) with U6 small nuclear RNA (snRNA) as endogenous control. StepOnePlus real-time PCR system (Applied Biosystems, Foster City, CA, USA) was used to carry out all PCR reactions. Reaction conditions were: $95{ }^{\circ} \mathrm{C}$ for $50 \mathrm{~s}$, followed by 40 cycles of $95{ }^{\circ} \mathrm{C}$ for $15 \mathrm{~s}$ and $58^{\circ} \mathrm{C}$ for $30 \mathrm{~s}$. This experiment was performed in triplicate manner and $\mathrm{Ct}$ values were analyzed using $2^{-\Delta \Delta \mathrm{CT}}$ method.

\section{Enzyme-linked immunosorbent assay (ELISA)}

Serum levels of IL-2, IL-17, IL-10, IL-19, IL-20 and IL-35 were measured by ELISA using corresponding ELISA kits. Human IL-2 (D2050), IL-10 (D1000B), IL17 (D1700), IL-19 (D1900) and IL-20 (DL200) ELISA kits were provided by R\&D Systems. Human IL35 ELISA Kit (LS-F27033) was provided by LSBio. All operations were performed in strict accordance with the kits' instructions.

\section{Statistical analysis}

All data were processed using Graphpad Prism 6 software. All data were expressed as mean \pm standard deviation and compared by unpaired t tests. ROC curve analysis was performed to evaluate the diagnostic value of miR 150-5p for generalized myasthenia gravis with generalized myasthenia gravis patients ast true positive cases and healthy controls as true negative cases. Correlation analyses were performed by Pearson correlation coefficient. $P<0.05$ was considered to be statistically significant.

\section{RESULTS}

Upregulation of miR150-5p distinguished generalized myasthenia gravis patients from healthy controls

Expression of miR150-5p in serum of both patients with generalized myasthenia gravis and healthy controls 
A

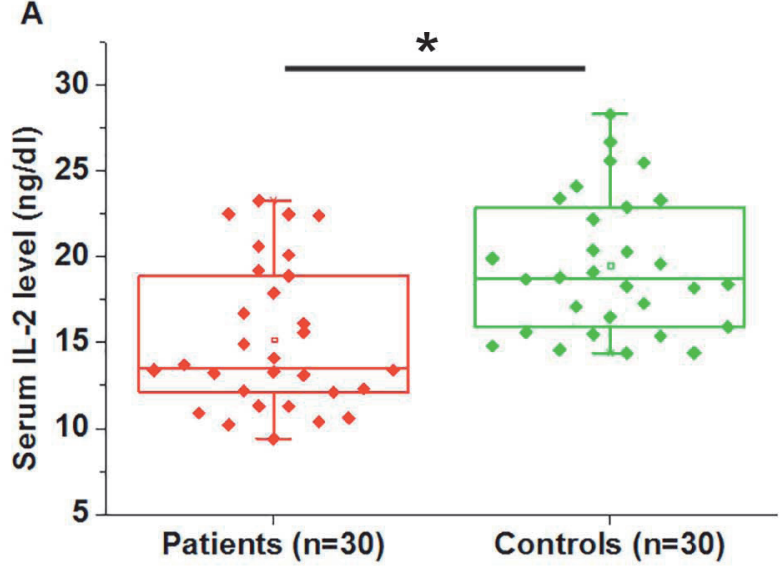

C

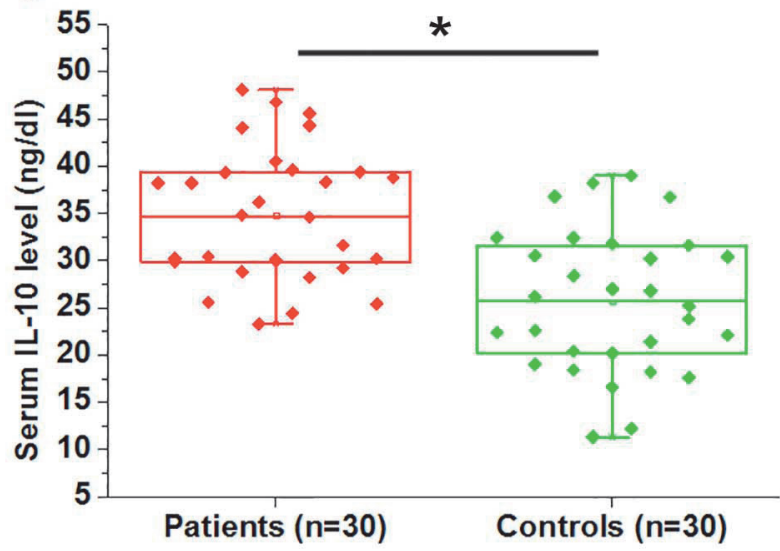

E

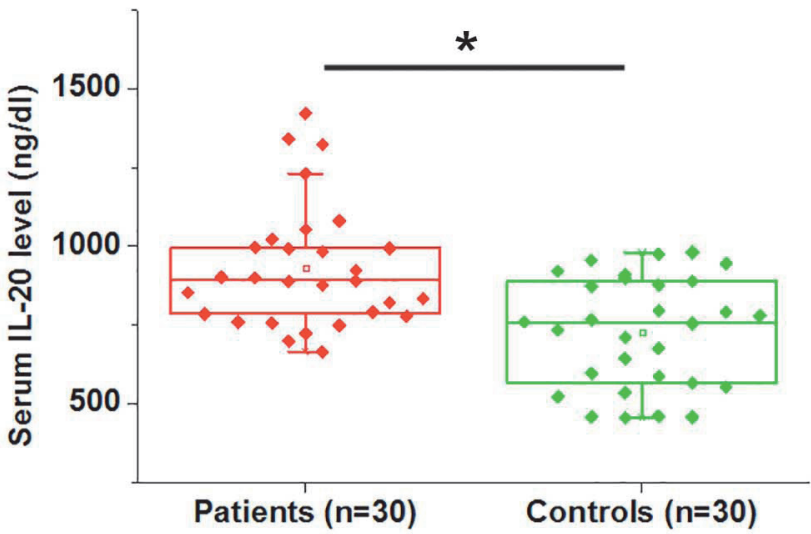

B

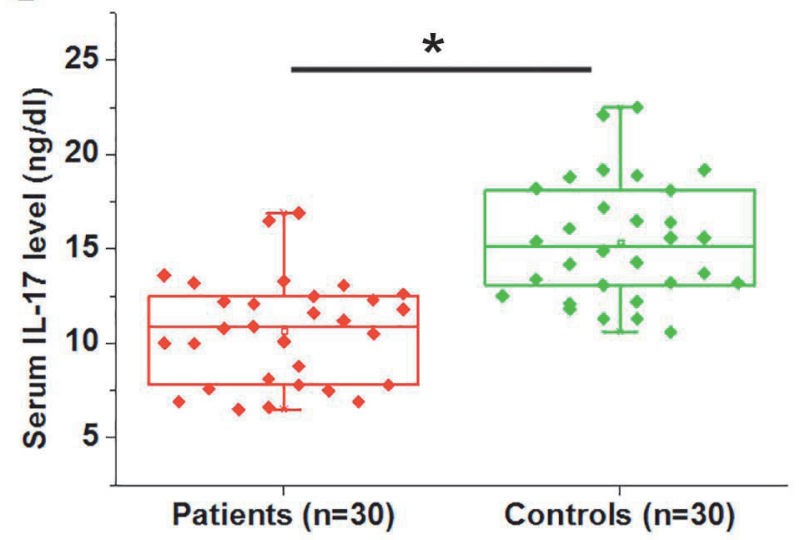

D

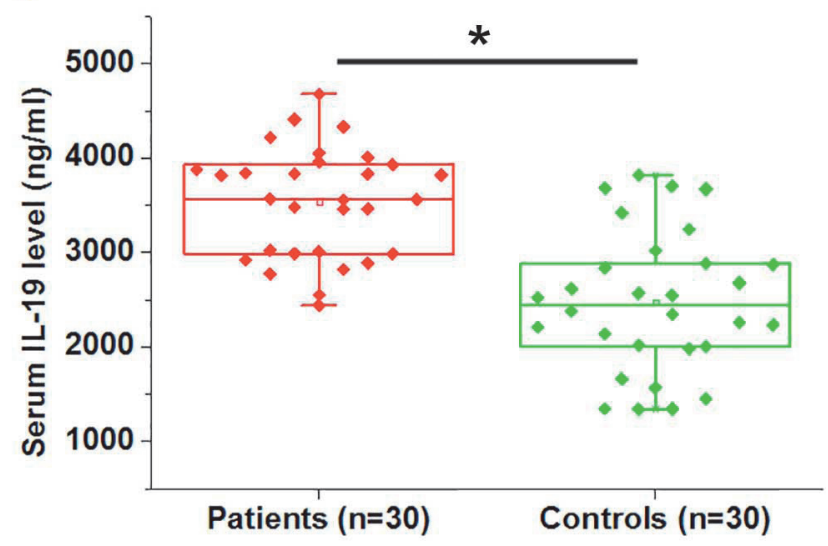

$\mathrm{F}$

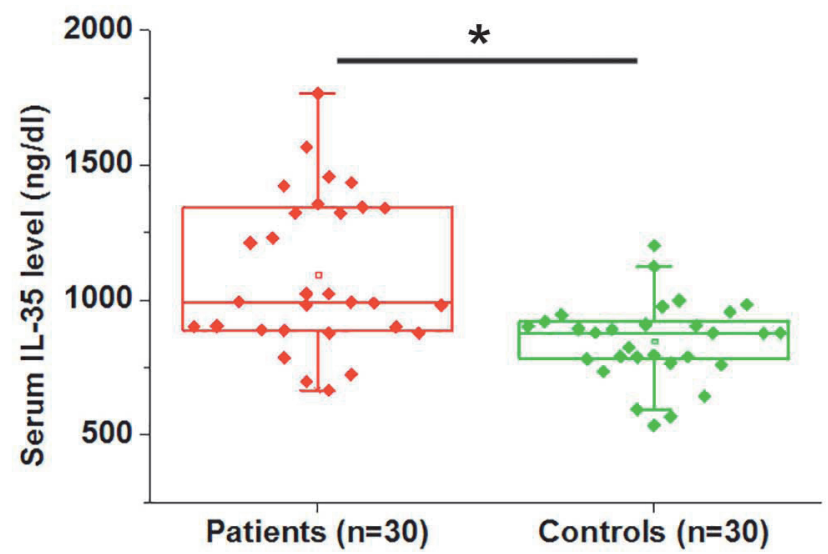

Fig. 2. Different cytokines show different expression patterns in patients with generalized myasthenia gravis.

Data here show the comparison of serum levels of IL-2 (A), IL-17 (B), IL-10 (C), IL-19 (D), IL-20 (E) and IL-35 (F) between patients with generalized myasthenia gravis and healthy controls Compared with healthy controls, significantly decreased serum levels of IL-2 and IL-17 and significantly increased serum levels of IL-10 IL-19, IL-20 and IL-35 were observed in patients with generalized myasthenia gravis $\left({ }^{*}, P<0.05\right)$.

was detected by qRT-PCR. Compared with healthy controls, expression of miR $150-5 p$ in serum was significantly upregulated in generalized myasthenia gravis patients (Fig. 1a, $P<0.05$ ). ROC curve analysis was performed to evaluate the diagnostic value of $\mathrm{miR} 150-5 \mathrm{p}$ for general- ized myasthenia gravis with generalized myasthenia gravis patients at true positive cases and healthy controls as true negative cases. As shown in Fig. 1b, area under the curve was 0.8617 , with standard error of 0.04532 and $95 \%$ confidence interval of [0.7730, 0.9503]. 
A

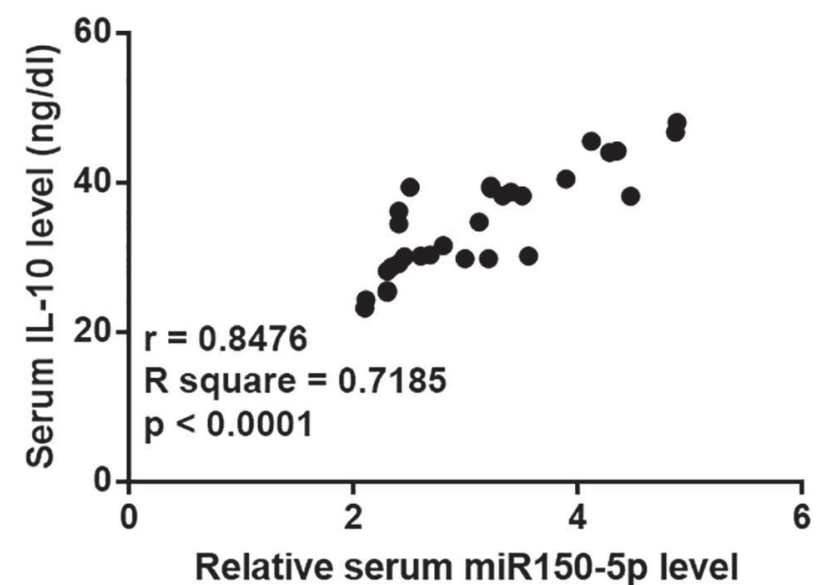

C

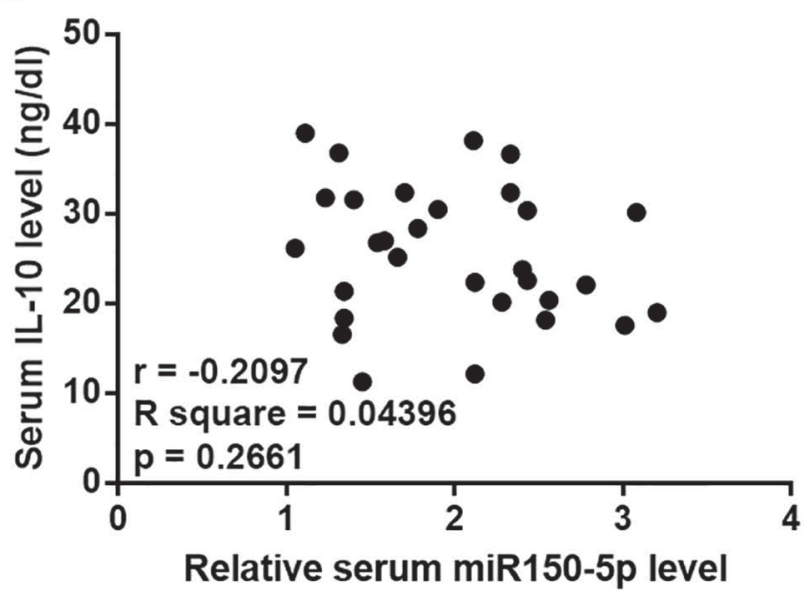

B

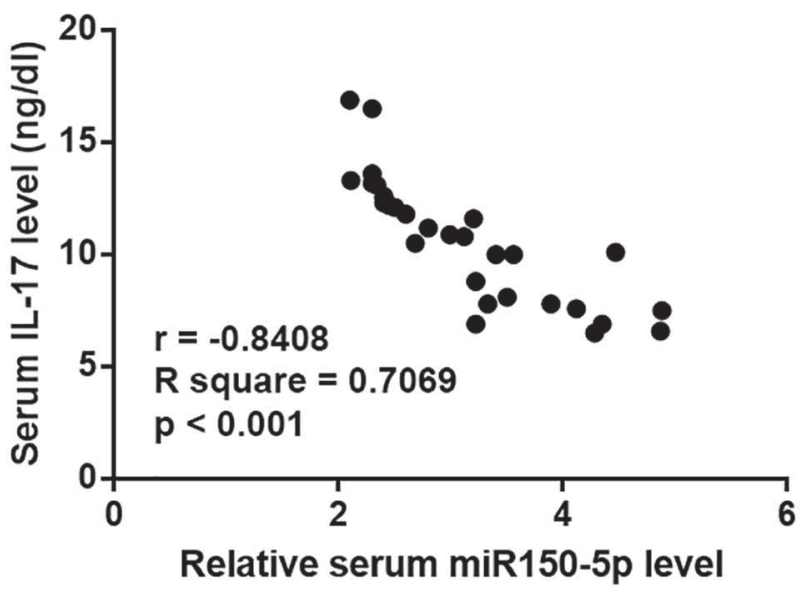

D

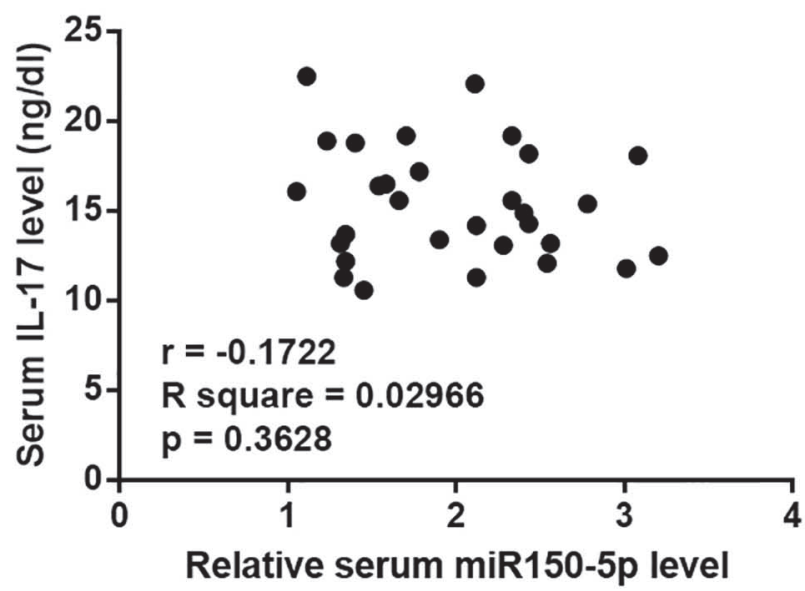

Fig. 3. Serum levels of miR150-5p were positively correlated with IL-10 and negatively correlated with IL-17.

Correlation analyses revealed a positive correlation between serum miR150-5p and IL-10 (A) and a negative correlation between serum miR150-5p and IL-10 (B). But miR150-5p was not significantly correlated with IL-10 (C) and IL-10 (D) in healthy controls. Here R square $>0.65$ indicated a strong correlation.

Different cytokines show different expression patterns in patients with generalized myasthenia gravis

Serum levels of IL-2, IL-17, IL-10, IL-19, IL-20 and IL35 were measured by ELISA using corresponding ELISA kits. Compared with healthy controls, significantly decreased serum levels of IL-2 (Fig. 2A) and IL-17 (Fig. 2B) and significantly increased serum levels of IL-10 (Fig. 2C), IL-19 (Fig. 2D), IL-20 (Fig. 2E) and IL-35 (Fig. 2F) were observed in patients with generalized myasthenia gravis $(P<0.05)$.

Serum levels of miR150-5p were positively correlated with IL-10 and negatively correlated with IL-17

Correlations between serum levels of miR $150-5 p$ and different serum cytokines in patients with generalized myasthenia gravis were analyzed by Pearson correlation coefficient. The results showed that serum IL-10 was significantly positively correlated with serum miR $150-5 p$ (Fig. 3A) and serum IL-17 was significantly negatively correlated with serum miR150-5p (Fig. 3B). However, miR $150-5 p$ was not significantly correlated with IL-10 (Fig. 3C) or IL-10 (Fig. 3D) in healthy controls. In ad- dition, no significant correlations were found between serum miR150-5p and other cytokines (data not shown).

Medical treatment led to decreased serum levels of miR150-5p and IL-10 as well as increased serum levels of IL-2 and IL-17

Compared with the pre-treatment levels, significantly decreased serum levels of miR150-5p (Fig. 4A) and IL-10 (Fig. 4B) as well as increased serum levels of IL-2 (Fig. 4C) and IL-17 (Fig. 4D) were observed after treatment in patients with generalized myasthenia gravis. No significant changes were found in other serum cytokines.

\section{DISCUSSION}

Myasthenia gravis can be divided into different subtypes, and the subtype of myasthenia gravis that affects multiple muscle groups in the human body is named generalized myasthenia gravis. Our study only included patients with generalized myasthenia gravis as subjects to reduce the study complexity. Our study further confirmed 
A
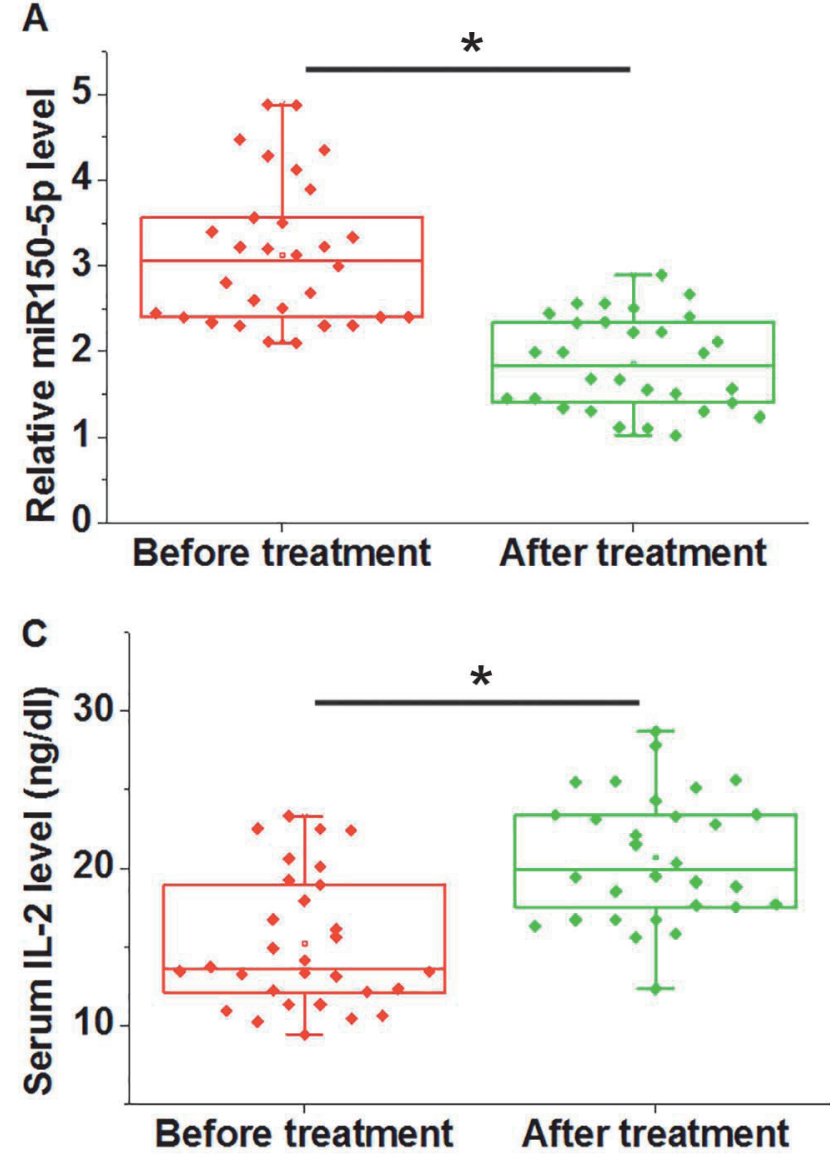

B
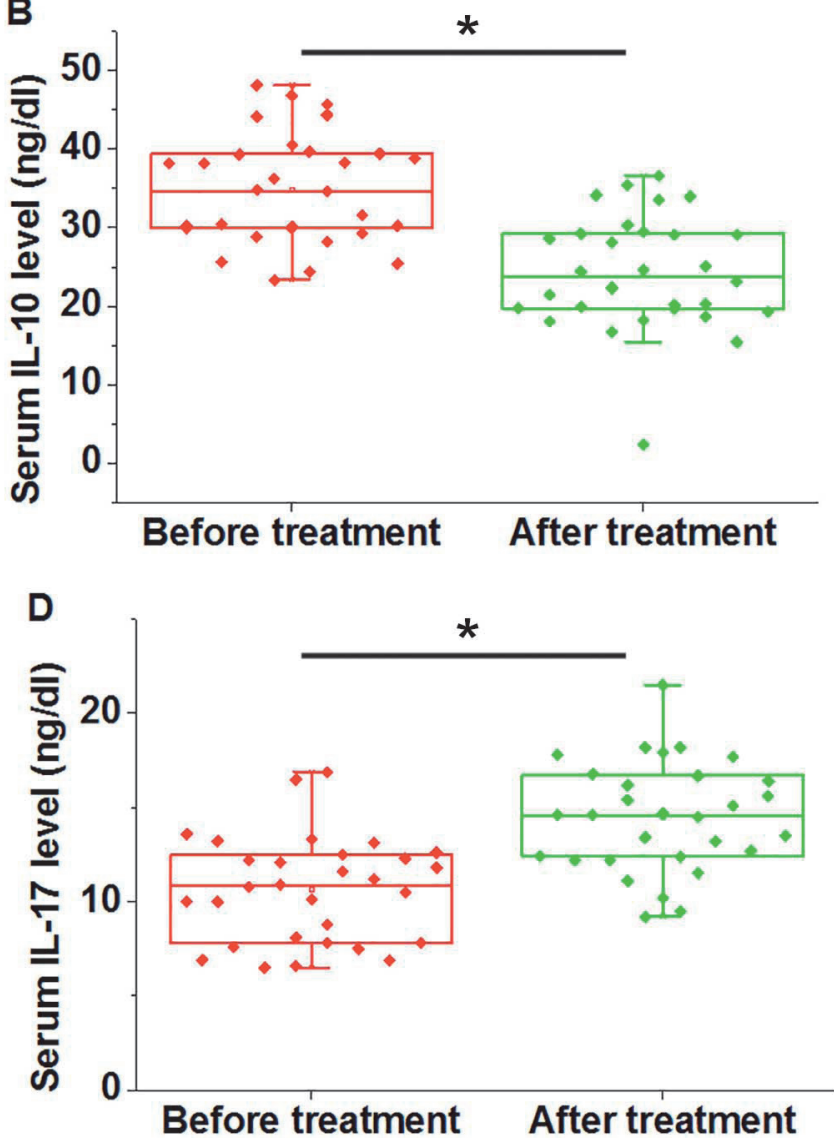

Fig. 4. Medical treatment led to decreased serum levels of miR150-5p and IL-10 as well as increased serum levels of IL-2 and IL-17. After treatment, ELISA results revealed decreased serum levels of miR150-5p (A) and IL-10 (B) as well as increased serum levels of IL-2 (C) and IL-17 (D) in patients with generalized myasthenia gravis (,$P<0.05)$.

the involvement of miR150-5p in myasthenia gravis. We also proved that the miR $150-5 \mathrm{p}$ may participate in the pathogenesis of myasthenia gravis through the interactions of inflammatory cytokines.

The onset, development and progression of myasthenia gravis require the involvement of multiple internal and external factors. However, it is generally believed that genetic factors play central role in the pathogenesis of this disease ${ }^{14}$. Development of myasthenia gravis also globally affects the expression of genes, including miRNAs ( ref. $^{15}$ ), indicating the involvement of miRNA in this disease. In effect, circulating miRNAs that show altered expression pattern in myasthenia gravis patients have proved to be with potentials in the diagnosis of this disease ${ }^{16}$. Altered expression of miR-150-5p has been observed in several types of human diseases, such as glioma ${ }^{17}$ and acute ischemic stroke ${ }^{18}$. In a recent study, Punga et al. showed that miR-150-5p is significantly upregulated in patients with myasthenia gravis comparing with healthy controls ${ }^{12}$. Consistent with this study, in our study, we also observed significantly higher serum levels of miR-150-5p in myasthenia gravis patients than those in healthy controls. In addition, upregulation of miR-150-5p effectively distinguished myasthenia gravis patients from healthy controls.
Our data further confirmed the involvement of miR-150$5 \mathrm{p}$ in myasthenia gravis.

As an autoimmune disease, myasthenia gravis also causes changes in expression patterns of anti- and proinflammatory cytokines ${ }^{19}$. Some therapeutic interventions, such as physical exercise have been proved to improve the signs of myasthenia gravis by improving the balance between anti- and pro-inflammatory cytokines ${ }^{20}$. However, physical exercise is not practical because most patients cannot tolerate physical exercise. In this study we observed decreased serum levels of IL-2 and IL-17 and increased serum levels of IL-10, IL-19, IL-20 and IL-35 in patients with generalized myasthenia gravis. However, correlation analysis only revealed a significantly positive correlation between IL-10 and miR150-5p and a significantly negative correlation between IL-17 and miR150$5 \mathrm{p}$. Therefore, miR150-5p may participate in myasthenia gravis through the interactions with IL-10 and IL-17 but not with other inflammatory cytokines. In addition, the interactions between IL-17, IL-10 and miR150-5p are indirect due to the lack of correlations between the healthy controls. Therefore, there should be disease-specific mediators between them. 


\section{CONCLUSION}

In this study we revealed that miR150-5p may participate in myasthenia gravis through the interactions with IL-10 and IL-17. miR150-5p is upregulated in patients with myasthenia gravis compared with healthy controls. Changes in expression levels of miR $150-5 p$ reflect the treatment outcomes of myasthenia gravis and miR150$5 \mathrm{p}$ may be used as a biomarker to guide the treatment of myasthenia gravis. Monitoring the changes of inflammatory cytokines and miR150-5p may help to improve treatment strategies.

Author contributions: WA, YL: experimental design; WA, CT: performing experiments; YH, WW: materials preparation; WA, XH, YL: data analysis; YL: manuscript writing; All authors: manuscript proofreading.

Conflict of interest statement: None declared.

\section{REFERENCES}

1. Gilhus NE, Verschuuren JJ. Myasthenia gravis: subgroup classification and therapeutic strategies. Lancet Neurol 2015;14(10):1023-36.

2. Westerberg E. Environmental Factors of Importance in Myasthenia Gravis. Emphasis on Physical Activity. Digital Comprehensive Summaries of Uppsala Dissertations from the Faculty of Medicine. Acta Universitatis Upsaliensis; 2018.

3. Meraouna A, Cizeron-Clairac G, Le Panse R, Bismuth J, Truffault F, Tallaksen C, Berrih-Aknin S. The chemokine CXCL13 is a key molecule in autoimmune myasthenia gravis. Blood 2006;108(2):432-40.

4. Cufi P, Dragin N, Ruhlmann N, Weiss JM, Fadel E, Serraf A, BerrihSknin S, Le Panse R. Central role of interferon-beta in thymic events leading to myasthenia gravis. J Autoimmun 2014;52:44-52.

5. Kanai T, Uzawa A, Kawaguchi N, Himuro K, Oda F, Ozawa Y, Kuwabara $\mathrm{S}$. Adequate tacrolimus concentration for myasthenia gravis treatment. Eur J Neurol 2017;24(2):270-5.

6. Iwakawa $\mathrm{H}$, Tomari Y. The functions of microRNAs: mRNA decay and translational repression. Trends Cell Biol 2015;25(11):651-65.

7. Bartel DP. MicroRNAs: genomics, biogenesis, mechanism, and function. Cell 2004;116(2):281-97.
8. Hammond SM. An overview of microRNAs. Adv Drug Deliv Rev 2015;87:3-14.

9. Wang J, Zheng S, Xin N, Dou C, Fu L, Zhang X, Chen J, Zhang $Y$ Geng D, Xiao C, Cui G, Shen X, Lu Y, Wang J, Dong R, Qiao Y, Zhang Y. Identification of novel MicroRNA signatures linked to experimental autoimmune myasthenia gravis pathogenesis: down-regulated miR145 promotes pathogenetic Th17 cell response. J Neuroimmune Pharmacol 2013;8(5):1287-302.

10. Uzawa A, Kanai T, Kawaguchi N, Oda F, Himuro K, Kuwabara S. Changes in inflammatory cytokine networks in myasthenia gravis. Sci Rep 2016;6:25886.

11. Berrih-Aknin S, Le Panse R. Myasthenia gravis: a comprehensive review of immune dysregulation and etiological mechanisms. J Autoimmun 2014;52:90-100.

12. Punga $T$, Le Panse $R$, Andersson $M$, Truffault $F$, Berrih-Aknin S, Punga AR. Circulating mi RNA s in myasthenia gravis: miR-150-5p as a new potential biomarker. Ann Clin Transl Neurol 2014;1(1):49-58.

13. Li Z Y. China guidelines for the diagnosis and treatment of generalized myasthenia gravis. Neurol Neuroimmunol Neuroinflamm 2016;3:1-9.

14. Avidan N, Le Panse R, Berrih-Aknin S, Miller A. Genetic basis of myasthenia gravis-a comprehensive review. J Autoimmun 2014;52:14653.

15. Nogales-Gadea G, Ramos-Fransi A, Suarez-Calvet X, Navas M, RojasGarcia R, Mosquera JL, Diaz-Manera J, Querol L, Gallardo E, Illa I. Analysis of serum miRNA profiles of myasthenia gravis patients. PLoS One 2014;9(3):e91927.

16. Punga A R, Punga T. Circulating microRNAs as potential biomarkers in myasthenia gravis patients. Ann N Y Acad Sci 2018;1412(1):33-40.

17. Sakr M, Takino T, Sabit H, Nakada M, Li Z, Sato H. miR-150-5p and miR-133a suppress glioma cell proliferation and migration through targeting membrane-type-1 matrix metalloproteinase. Gene 2016;587(2):155-62.

18. Scherrer N, Fays F, Mueller B, Luft A, Fluri F, Christ-Crain M, Devaux Y, Katan M. MicroRNA 150-5p improves risk classification for mortality within 90 days after acute ischemic stroke. J Stroke 2017;19(3):32332.

19. Yilmaz V, Oflazer P, Aysal F, Durmus H, Poulas K, Yentur SP, GulsenParman Y, Tzartos S, Marx A, Tuzun E, Deymeer F, Saruhan-Direskeneli G. Differential cytokine changes in patients with myasthenia gravis with antibodies against AChR and MuSK. PloS One 2015;10(4):e0123546.

20. Westerberg E, Molin C J, Lindblad I, Emtner M, Punga AR. Physical exercise in myasthenia gravis is safe and improves neuromuscular parameters and physical performance-based measures: A pilot study. Muscle Nerve 2017;56(2):207-14 